\title{
Caracterización de la Cadena de Comercialización de la Langosta (Jasus Frontalis) del Archipiélago Juan Fernández
}

\author{
Luis Sáez T. ${ }^{1}$; Gianinna Adofacci G. ${ }^{1}$; Mario Maino M. ${ }^{2}$ \\ ${ }^{1}$ Departamento de Gestión Agraria, Universidad de Santiago de Chile \\ ${ }^{2}$ Facultad de Ciencias Veterinarias y Pecuarias, Universidad De Chile \\ Avenida Ecuador, 3769, Estación Central, Santiago, Chile. Email: luis.saez@usach.cl
}

\begin{abstract}
Resumen
La economía del Archipiélago Juan Fernández, se sustenta principalmente por la extracción y comercialización de las langostas vivas y, en menor medida, por la venta de otras especies. Los pescadores de la zona, en forma individual o en varias agrupaciones, venden a empresas intermediarias establecidas en las islas Robinson Crusoe y Alejandro Selkirk, o bien, se comprometen a entregar el recurso en forma exclusiva a algún comprador, mediante convenios informales.

La presente investigación, describe la estructura comercial de la langosta (Jasus Frontalis), del archipiélago de Juan Fernández, describiendo todos los integrantes de su cadena comercial, de la cual no habían precedentes, se estiman los costos y márgenes de comercialización, para cada eslabón, y finalmente se realiza un análisis de costos de los pescadores, otorgando antecedentes que permitan generar estrategias de corto, mediano y largo plazo, con el fin de lograr, una comercialización más eficiente, para el primer eslabón de la cadena.

Esta investigación es un estudio de tipo exploratorio, realizado entre los años 2007 y 2008., cuyos resultados permiten conocer los principales actores que participan en la cadena de comercialización de las langostas, estos son: Pescadores, Intermediarios en la Isla y del Continente. Se determinó que, los pescadores obtienen un 37,9\% de retorno sobre sus ventas, por lo que son el eslabón más rentable en relación a los costos. Sin embargo el margen de comercialización indica que los pescadores ganan el $18,7 \%$ del precio final.
\end{abstract}

Palabras claves: Comercialización, Cadena, Costos, Langostas, Archipiélago de Juan Fernández, Pescadores

\section{Introducción}

En el Archipiélago Juan Fernández habitan aproximadamente 600 personas, de las cuales la gran mayoría se dedican a la pesca artesanal y extracción de la langosta, siendo esta última, la que les aporta más ingresos.

El pueblo habita principalmente en la Isla Robinson Crusoe, trasladándose algunos de ellos en temporada de langosta a la de Alejandro Selkin, por el flujo mayor de la extracción (Arana, 2000). El Archipiélago Juan Fernández, tiene un rustico desarrollo urbano, las comodidades y la tecnología de la modernidad, han ido llegando lentamente. Las personas, la mayoría pescadores, tienen grandes necesidades de salud y educación, dificultando su calidad de vida.

En este archipiélago existe una abundante flora y fauna marina, caracterizada por la presencia de numerosos crustáceos, peces, octópodos, moluscos, entre muchos otros. Ahora bien desde el punto de vista de su descripción en faenas pesqueras, las más importantes son la langosta (Jasus frontalis), el cangrejo dorado (Chaceon chilensis), la centolla (Paromola rathbuni), la jaiba remadadora (Ovalipes trimaculatus), y la langosta chata (Scyllarus delfini). (Andrade, 1985)

Los cuales son muy apreciados por la calidad de sus carnes y sabores, que no se encuentran en la zona costera continental. Desde fines del siglo XIX, en el Archipiélago de Juan Fernández, se inicia la 
explotación de la langosta, en forma artesanal y sostenida, recurso marino de gran importancia socioeconómica, ya que su extracción constituye la principal actividad realizada por los habitantes del archipiélago, además de representar el $98 \%$ de las capturas nacionales de langosta.

La comercialización de los productos marinos del archipiélago, en especial el recurso de la langosta, podría considerarse como estratégico y prioritario, ya que la actividad productiva industrial, es prácticamente nula, lo que genera que la pesca extractiva sea fundamental, para la economía de la isla, por tanto la potencialización de ésta actividad, podría generar mejores condiciones, y más recursos a la zona.

De todos los recursos pesqueros de Juan Fernández, la langosta es la que alcanza mayor valor en el mercado, aunado a su abundancia y amplia distribución, la han convertido en la principal fuente de ingresos para la comunidad. La experiencia internacional, como la del caribe Mesoamericano, que comprende las costas de Honduras, Guatemala, Belice y México habla que la producción promedio anual de langosta entera es de unas 40.000 toneladas métricas, con un valor aproximado en playa de 350 millones de dólares estadounidenses. Este valor se puede incrementar hasta en un $300 \%$ cuando la langosta llega al consumidor final. Situación similar, ocurre con la langosta de Juan Fernández.

El objetivo del presente trabajo es describir la cadena de comercialización de la langosta (Jasus frontalis) del archipiélago de Juan Fernández, caracterizando a los principales actores, que participan en la cadena; estimando los costos y márgenes de comercialización involucrados, y los costos relevantes que influyen en las utilidades de los pescadores

\section{Metodología}

Se realizaron entrevistas a agentes claves, como dirigentes del Sindicato de pescadores, y a autoridades del Servicio Nacional de Pesca (SERNAPESCA), antecedentes utilizados para diseñar sendos cuestionarios, aplicados en forma presencial a todos los pescadores dueños de embarcaciones e intermediarios de esta cadena, es decir, se realizó censo.

Los costos, y márgenes de comercialización involucrados, a lo largo de la cadena de comercialización, se estimaron en posiciones relevantes del mercado, como lo es el mercado playa, intermediario, continente, exportación. Considerando, en la primera etapa, todos los costos e ingresos que ocurren hasta la primera venta. En ambos casos, se consideraron los promedios, por cada grupo de pescadores, además de calcular la desviación estándar, para cada ítem de costo involucrado a través de la cadena, con el fin de identificar los rangos en los que varían estos datos.

Posteriormente en la segunda etapa, se estimaron los costos relacionados con la venta del producto postcaptura, considerando por ejemplo, los costos de transporte a los mercados de destino, y los ingresos obtenidos en la venta de estos, los cuales se expresaron en Euro, para la comercialización internacional, debido a que la langosta (Jasus frontalis) es demandada en el mercado europeo, principalmente Madrid, Italia, entre otros; y en pesos, en el mercado nacional, tomando como años bases la temporada 2007- 2008.

Con los ingresos y costos de cada etapa, se determinaron los flujos financieros generados por la explotación de la langosta, los cuales también fueron calculados, para cada eslabón de la cadena, con el objetivo de determinar si existen distintas utilidades y márgenes por agrupaciones de pescadores según intermediarios.

El ingreso total, generado por la embarcación, se calculó mediante la multiplicación entre el desembarque promedio de cada mes, por temporada, y el precio registrado. Para la estimación de los ingresos generados por la actividad, se tomaron precios de venta en playa, durante la temporada, como también de temporadas anteriores, con el fin de poder visualizar las variaciones que se ha tenido durante la temporada del 2001 hasta el 2008.

Tratándose de un producto fresco, donde los atributos esenciales es que se comercialice vivo, se debe tomar en cuenta la cantidad efectiva de las langostas que llega al mercado destino. Por lo tanto es fundamental considerar el porcentaje de mortalidad de ésta especie, según los datos arrojados por los intermediarios, donde se consideraron los porcentajes de mortalidad en; transporte al continente, los viveros y transporte a mercado destino.

Posteriormente, se realizó un análisis de los costos variables por trampa de cada pescador en una temporada con el fin de identificar qué insumos, al aumentar sus costos, tenían mayor incidencia en las utilidades de los pescadores, mediante el programa 
estadístico "Paquete Estadístico para las Ciencias Sociales"(SPSS), se verificó la normalidad de los datos, aplicando el test de Kolmogorov-Smirnov, luego se procedió a medir el grado de asociación lineal entre dos variables, a través del Coeficiente de Correlación de Pearson.

\section{Resultados y Discusión}

Aspectos generales de la comercialización

La cadena de comercialización comienza con los pescadores, quienes venden en playa sus capturas. Se identificaron tres intermediarios, quienes concentran el $82 \%$ de las langostas transportadas, la diferencia, se vende de manera directa al único intermediario del continente. En general, el $90 \%$ de las langostas de envían al intermediario del continente.

Los dueños de botes, venden a alguno de los tres intermediarios de la isla, quienes, además de comprar la producción, proveen de insumos a los pescadores. Un $25 \%$ de los dueños de botes le vende su producción al denominado Intermediario 1 ; el $30 \%$ le venden al Intermediario $2 \mathrm{y}$, el $27 \%$ venden su captura al Intermediario 3, que corresponde al Sindicato de pescadores de la isla.
Selkirk, sólo hay 13 pescadores. Esta actividad se ha realizado a través de varias generaciones, heredando los bienes, y trasmitiendo su experiencia de padres a hijos. El nivel de escolaridad en la mayoría de ellos, es enseñanza básica cursada, sólo el $2 \%$ tiene el ciclo completo. En cuanto a características etáreas, más del $50 \%$ de ellos se encuentra bajo los 50 años.

Según los datos acerca de las embarcaciones, recopilados por la encuesta, estas tienen un número de trampas promedio de 32, con una desviación estándar de 8,4. En cuanto, al número de marcas - Definición de marcas: lugares establecidos, en la Bahia Cumberlan, en los que cada pescador autorizado, localiza sus trampas langosteras - por bote, el promedio es de 95, con una desviación estándar de 69 , lo que se explica, mediante el sistema del traspaso de las marcas, por generaciones.

Intermediarios de Robinson Crusoe. Corresponden a micro empresarios locales, que se dedican a comprar la langostas a los pescadores, las que almacenan en sus viveros, las embalan y envían al continente En este eslabón hay tres participantes, cuya escolaridad esta dentro del rango superior incompleta y media completa. En cuanto a las edades están entre los 40 a 60 años de edad. Cada intermediario tiene en promedio 5 viveros - Vivero: son recipiente donde los pescadores guardan sus langostas, ubicados en el fondo del mar -,

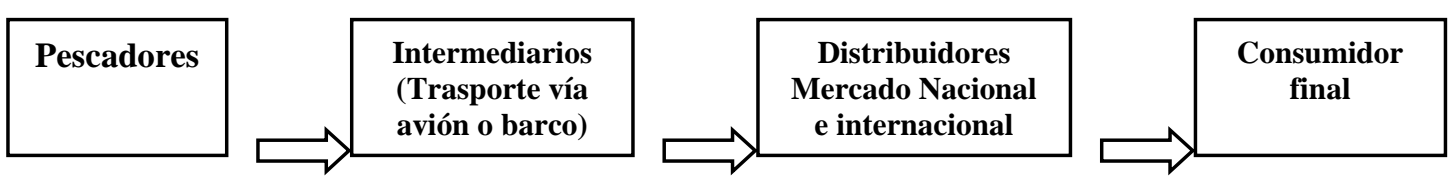

Figura 1: Integrantes de la Cadena de comercialización de la langosta (Jasus frontalis)

Fuente: Elaboración propia en base a la información de las encuestas.

A continuación, se caracterizan a cada uno de los integrantes de la cadena, según aspectos socioculturales.

Pescadores: Dado que la principal actividad económica, en las islas del Archipiélago Juan Fernández, es la pesca, la mayor parte de la fuerza laboral local, está relacionada con ella. Según los resultados, la mayoría de las familias, cuentan con uno o más de sus miembros dedicado a estas faenas. Se ha determinado que en la isla Robinson Crusoe, laboran 76 pescadores, y existen 38 botes, donde trabajan 2 personas por embarcación, de los cuales el 93\%, se encuentra inscrito en el sindicato. En la isla Alejandro y cuentan con una mano de obra de dos personas.

Distribuidores o intermediario (Continente). Las personas que componen éste eslabón de la cadena, se encargan de retirar y almacenar en sus viveros el producto, para posteriormente comercializar el $98 \%$ de las langosta, en el mercado internacional, y el resto en el mercado nacional, como a restaurantes, hoteles, entre otros. Se encuentran, en el rango de escolaridad superior completa, y un rango de edad entre 30 y 70 años. Según los datos obtenidos por la entrevista, arriendan los servicios de viveros a la Universidad Católica de Valparaíso y al Centro de Quintay de la Universidad Andrés Bello. 
Dentro de esta cadena de comercialización se refleja un sistema circular en cuanto a proveedor - pescador intermediario isla - intermediario continente, explicándose a través de tres causas importantes para los pescadores, la primera es la necesidad de disponer capital de trabajo para salir a la faena de pesca, lo cual conlleva a recurrir a las empresas intermediarias que financien sus salidas haciendo un compromiso de venta exclusiva de su captura, la segunda es debido a que existe una veda del recurso, las empresas intermediarias proveen créditos para solventar los gastos de reparación y mantención de las embarcaciones y aparejos de pesca, comprometiendo nuevamente la producción de la temporada siguiente. Y finalmente la gran distancia con el mercado consumidor, ya que actualmente el pescador para obtener un buen precio por su producción, debido a que sus costos no se reducirán a través de los beneficios de las empresas intermediarias. Esta situación entorpece y encarecen los costos de insumos, a la primera venta, donde quedo demostrado que los mas perjudicados son los pescadores del grupo I1, obteniendo un retorno sobre sus ventas de $3,7 \%$ siendo los menos eficientes.
Luego de caracterizar a los distintos eslabones de la cadena de comercialización, se presenta la estimación de todos los costos involucrados a lo largo de la cadena. Esta valoración se considera en dos etapas; en la primera etapa, se estiman todos los costos relacionados hasta la venta en playa, y en la segunda etapa, todos los costos hasta el mercado internacional.

\section{PRIMERA ETAPA:}

La estimación de costos, en la primera etapa de comercialización, se incluyó los costos involucrados desde la extracción hasta la primera venta, es decir, mercado playa. Estos son:

Inversión: Son los aquellos que se incurren, en la embarcación, con la que se realiza la faena de pesca, equipada completa (motor, bote, virador o chigre), su valor promedio $\$ 5.602 .500$.

Costos de mantención: Son aquellos, que se destinan a mantener en buen estado la embarcación, se presentan en Tabla 1.

Tabla 1: Cantidad de insumos utilizados para mantención

\begin{tabular}{|c|c|c|c|c|c|}
\hline Insumos & $\begin{array}{l}\text { Cantidad por } \\
\text { temporada }\end{array}$ & $\begin{array}{l}\text { Promedio por } \\
\text { temporada, por } \\
\text { embarcación }\end{array}$ & $\begin{array}{l}\text { Cantidad } \\
\text { mensual }\end{array}$ & $\begin{array}{l}\text { Promedio } \\
\text { mensual, por } \\
\text { embarcación }\end{array}$ & $\begin{array}{l}\text { Desviación } \\
\text { Estándar } \\
\text { por } \\
\text { embarcación }\end{array}$ \\
\hline Varillas & 1.088 & $\$ 119.680$ & 136 & $\$ 14.960$ & $\$ 9.466$ \\
\hline Clavos & 16 kilos & $\$ 23.920$ & 2 kilos & $\$ 2.990$ & $\$ 5.772$ \\
\hline $\begin{array}{l}\text { Pintura } \\
\text { antialgas }\end{array}$ & 1 tarro & $\$ 28.000$ & 0,125 tarro & $\$ 3.500$ & $\$ 622$ \\
\hline Tiza & 5 kilos & $\$ 8.160$ & 0,625 kilos & $\$ 1020$ & $\$ 1.679$ \\
\hline $\begin{array}{l}\text { Listones de } \\
\text { Eucaliptos }\end{array}$ & 208 & 208 & 26 & $\$ 3.120$ & $\$ 2.302$ \\
\hline $\begin{array}{l}\text { Set de } \\
\text { herramientas }\end{array}$ & 1 & 1 & 0,125 & $\$ 2.064$ & $\$ 1.479$ \\
\hline Pabilo & 3 & 3 & 0,375 & $\$ 3.226$ & $\$ 3.597$ \\
\hline Otros & 1 & 1 & 0,125 & $\$ 5.322$ & $\$ 3.214$ \\
\hline Total & & & & $\$ 36.202$ & $\$ 28.131$ \\
\hline
\end{tabular}

Fuente: Elaboración propia en base a la información de las encuesta

Costos de insumos corrientes. Son aquellos costos en insumos necesarios para realizar la actividad de captura de langosta. El dato considerado, es el promedio mensual, de los gastos, según se expone en Tabla 2. 
Tabla 2. Cantidad por insumo de pesca en cada temporada

\begin{tabular}{lllllll}
\hline Insumos & $\begin{array}{l}\text { Cantidad } \\
\text { temporada }\end{array}$ & $\begin{array}{c}\text { por } \\
\text { Bencina }\end{array}$ & $\begin{array}{l}\text { Promedio } \\
\text { por } \\
\text { temporada }\end{array}$ & Cantidad mensual & $\begin{array}{l}\text { Promedio } \\
\text { mensual }\end{array}$ & $\begin{array}{l}\text { Desviación } \\
\text { Estándar }\end{array}$ \\
\hline Aceite & 3 bidones $(16001)$ & $\$ 1.315 .192$ & 1 tambor $(2001)$ & $\$ 164.399$ & $\$ 39.540$ \\
\hline Bujías & 4 & $\$ 41.432$ & 1,51 & $\$ 5.179$ & $\$ 4.667$ \\
\hline Filtros & 2 & $\$ 15.680$ & 0,625 & $\$ 2.450$ & $\$ \mathbf{1 . 6 4 3}$ \\
\hline Total & & $\$ 5.688$ & 0,25 & $\$ 711$ & $\$ \mathbf{2 . 4 8 9}$ \\
\hline
\end{tabular}

Fuente: Elaboración propia en base a la información de las encuestas.

Costos de insumos de pesca:

Se presentan a continuación, los costos según el lugar de compra (ver Tabla 3 y 4).

Tabla 3: Valores de los insumos, según el lugar de compra:

\begin{tabular}{lllll}
\hline Insumos & I1 & I2 & I3 & $\mathbf{D}$ \\
\hline Cordel & $\$ 23.350$ & $\$ 25.455$ & $\$ 19.900$ & $\mathbf{\$ 2 1 . 0 0 0}$ \\
Boyas & $\$ 54.000$ & $\$ 37.555$ & $\$ 33.300$ & $\mathbf{\$ 3 8 . 5 7 1}$ \\
Anzuelo & $\$ 1.860$ & $\$ 2.000$ & $\$ 1.850$ & $\mathbf{\$ 1 7 0 0}$ \\
Nylon & $\$ 2.250$ & $\$ 2.345$ & $\$ 2.220$ & $\mathbf{\$ 1 . 9 8 0}$ \\
Otros & $\$ 10.183$ & $\$ 8.419$ & $\$ 7.159$ & $\mathbf{\$ 7 . 8 6 9}$ \\
\hline Total & $\$ 91.643$ & $\$ 75.774$ & $\mathbf{\$ 6 4 . 4 2 9}$ & $\mathbf{\$ 7 1 . 1 2 0}$ \\
\hline
\end{tabular}

Fuente: Elaboración propia en base a la información de las encuesta.

Costos Totales: Los costos totales, en base a promedios mensuales, se consideraron la suma de los insumos de: mantención, corrientes y de pesca, resultando \$284.266.

Tabla 4: Cantidad de insumos mensuales y por temporada.

\begin{tabular}{llllll}
\hline Insumos & $\begin{array}{l}\text { Cantidad por } \\
\text { temporada }\end{array}$ & $\begin{array}{l}\text { Costos } \\
\text { promedios } \\
\text { por }\end{array}$ & $\begin{array}{l}\text { Cantidad } \\
\text { mensual } \\
\text { temporada }\end{array}$ & $\begin{array}{l}\text { Costo } \\
\text { Promedio } \\
\text { mensual }\end{array}$ & $\begin{array}{l}\text { Desviación } \\
\text { Estándar }\end{array}$ \\
\hline Cordel & 4 rollos & $\$ 190.384$ & 0,5 & $\$ 23.798$ & $\$ \mathbf{1 0 . 3 8 2}$ \\
\hline Boyas & $144 \mathrm{c} / \mathrm{u}$ & $\$ 301.544$ & 18 & $\$ 37.693$ & $\$ 27.571$ \\
\hline Anzuelo & $400 \mathrm{c} / \mathrm{u}$ & $\$ 6.860$ & 50 & $\$ 1.715$ & $\mathbf{\$ 1 . 3 6 6}$ \\
\hline Nylon & $480 \mathrm{~m}$ & $\$ 16.952$ & $60 \mathrm{~m}$. & $\$ 2.119$ & $\mathbf{\$ 2 . 0 8 9}$ \\
\hline Otros & 8 & $\$ 80.000$ & 1 & $\$ 10.000$ & $\mathbf{\$ 4 . 1 2 3}$ \\
\hline Total & & $\$ \mathbf{5 9 5 . 7 4 0}$ & & $\$ 75.325$ & $\mathbf{4 4 5 . 5 3 1}$ \\
\hline
\end{tabular}

Fuente: Elaboración propia en base a la información de las encuesta

\section{Ingresos Totales}

El ingreso total por bote, promedio por temporada, alcanzó un monto de $\$ 5.782 .000$, considerando que la temporada esta compuesta por 8 meses, se calcula un ingreso mensual promedio de $\$ 722.790$ por bote. El precio, de la langosta fue de $\$ 8.000$, con una captura total promedio por bote de 723 unidades.

Febrero y octubre, son los meses de mayor captura y comercialización de langostas (ver figura 2), generando en Febrero un ingreso promedio por 
embarcación de \$1.016.000, y en Octubre \$ 984.000. El mes que registro menor captura de las langostas, fue
Mayo, con un ingreso promedio de \$248.000.

Figura 2: Ingreso mensual promedio por bote, de la extracción de langostas (Jasus frontales) durante la temporada 2007/08 en Robinsón Crusoe

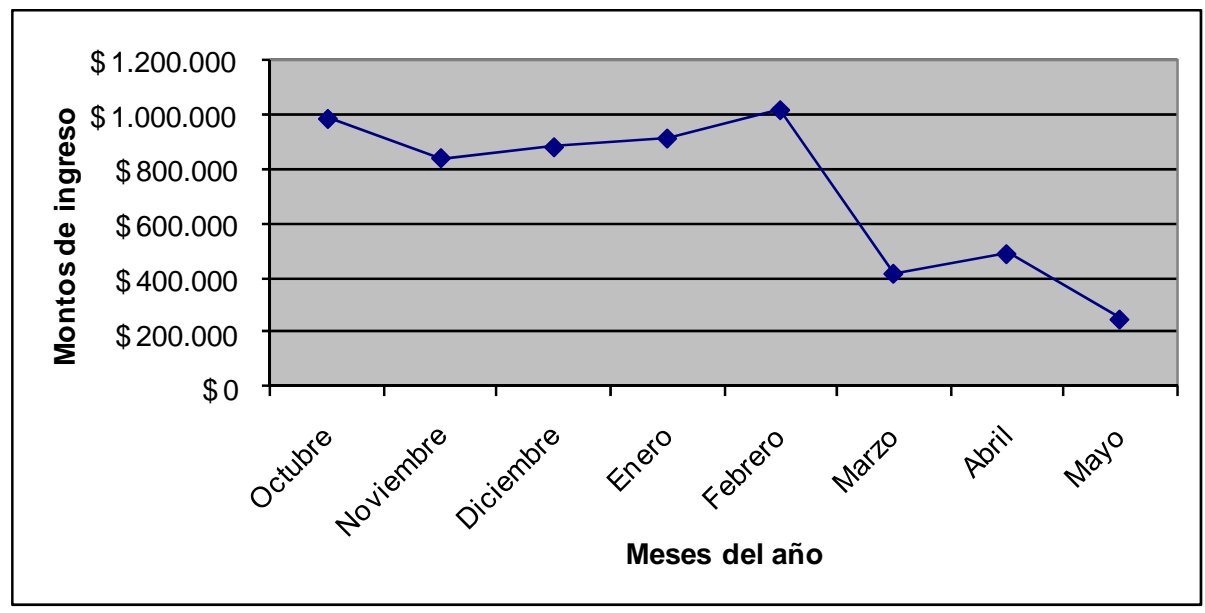

Fuente: Elaboración propia en base a la información de las encuestas.

Históricamente el precio de la langosta, ha sido manejado, por los intermediarios de la isla., por lo que es llamativo, que según el análisis de precios, en temporadas pasadas éste ha ido en alza. En la Figura 3, se muestra la curva en ascenso, de los precios nominales por temporada, según declaraciones en las encuestas.

Figura 3: Precio la langosta (Jasus Frontalis) en Robinson Crusoe, según temporada

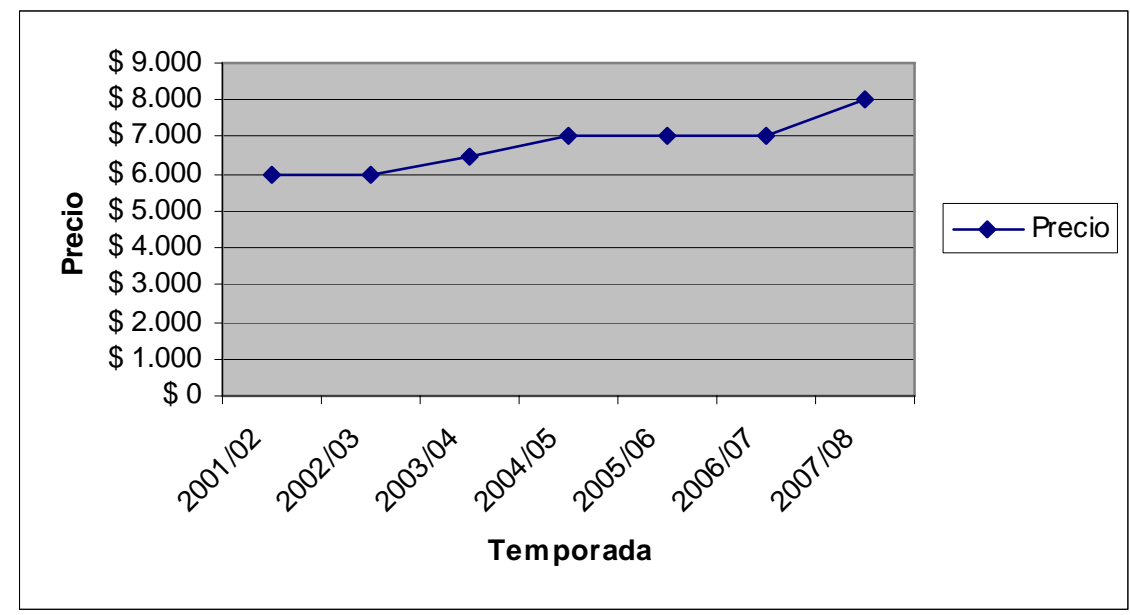

Fuente: Elaboración propia en base a la información de las encuestas

\section{SEGUNDA ETAPA:}

Costos según venta:

En esta etapa se consideraron los costos, en pesos, según la venta en el mercado nacional, (valor por unidad), e internacional, valor por kilo. 
Costos de mantención en viveros e instalaciones utilizadas:

Se consideraron todos los equipos, utilizados después de la primera venta, debido a la importancia, de la mantención de las langostas en viveros, puesto que después del viaje, no están en óptimas condiciones para ser comercializadas, por lo que deben "desestresarse" en los viveros. Por lo demás, los equipos tecnológicos utilizados para conservar las cualidades naturales de la langosta, deben encontrarse en buenas condiciones, alcanzando un valor de $\$ 500$ por cada langosta y $\$ 676$ por kilo.

Costo materia prima:

Las langostas en el mercado playa durante la temporada $2007-08$, tuvieron el valor de $\$ 8.000$ por unidad y $\$ 10.811$ el kilo.

Costos de transporte:

Estos costos variables están divididos en tres ítems:

a) Transporte Isla a Continente : valorados por vía aérea, ya que es el medio más utilizado para el envío de langostas, desde isla a continente, se contrata un viaje especial denominado Charter - Charter, es el arriendo de una avioneta, que permite transportar 1.000 ejemplares de Langostas desde el Archipiélago de Juan Fernández, por viaje. El precio que se indica, es el que cuesta el arriendo de la avioneta - donde una avioneta permite transportar 1.000 ejemplares por viaje El valor del transporte por unidad es de $\$ 1.800$ y por el Charter completo de $\$ 1.500 .000$.

b) Transporte de trayectos: El costo del transporte de trayectos, considera todos los recorridos que se realizan desde que la langosta llega al continente hasta el aeropuerto de embarque internacional, tales como: traslado de las langostas desde el aeródromo hasta los viveros, desde viveros a la planta certificadora y de planta certificadora al aeropuerto internacional, teniendo un valor de \$200 por unidad y \$ 270 por kilo. Resultando un total de $\$ 600$ por unidad y $\$ 810$ por kilo.

c) Transporte al mercado destino: El 98\% de las cantidades extraídas se exportan al mercado europeo, con un costo de envío que varía por peso de carga Extraído el 10 de Diciembre del 2008, de la fuente www.publitecsa.cl -.

Tabla 5: Costo del transporte langosta al mercado europeo (\$) - Un dólar equivale a \$ 504, se calculo basándose en su valor 2007/08 -.

\begin{tabular}{llll}
\hline Menos de 100 kilos & Entre 100-300 kilos & Entre 300-500 kilos & Mas de 500 kilos \\
\hline$\$ 1.512$ & $\$ 1.159,2-1.260$ & $\$ 1.008-1.159,2$ & $\$ 907,2-1.008$ \\
\hline
\end{tabular}

Fuente: Elaboración propia en base a la información de las encuestas.

Costos de Certificación PAC: En cuanto al costo de la certificación PAC, este fue es de $\$ 1.400$, por cada unidad, y $\$ 1.892$, por cada kilo. Este ítem lo deben pagar las personas que venden al extranjero, ya que es un programa de aseguramiento de calidad para productos pesqueros, el cual es exigido por el mercado Europeo.

Costos materiales de embalaje: El costo de materiales de embalaje se estimó en $\$ 400$ por unidad y $\$ 541$ por kilogramo.

Costo de Mano de Obra: Para esta actividad son necesarios 2 empleados y un administrador, este mismo personal realiza el embalaje. En cuanto a la mano de obra, mensualmente se destinan \$918.000, y $\$ 7.344 .000$, durante la temporada.
Agencia de Aduana - Cargo: Trámites de exportación y visa de exportación de Sernapesca, se destina un total de $\$ 200.000$.

Ingresos

En esta segunda etapa, exportación de langostas al mercado Europeo, los ingresos estimados están asociados a la cantidad efectiva de ejemplares que logran llegar a los mercados de destino, por lo se considera la mortalidad de la especie. De esta forma, al multiplicar la cantidad vendida por bote (723) y el número de botes (38), resultan 27.474 ejemplares. Considerando que la mortalidad en los viveros es un $2 \%$, por traslado al continente vía aérea es de $4 \%$ y la mortalidad en traslado a mercado de destino es de 
$0,04 \%$, se estima que las cantidades efectivas a vender al mercado internacional, ascienden a 25.815 ejemplares $\mathrm{y}$, al considerar un peso promedio de 740 gramos por unidad, equivale a 19.103 kilos (para exportación) a precio FOB promedio de 35 Euros $/ \mathrm{kg}$ (varía entre 32 y 38 euros el kilo - Extraído el 31 de Diciembre del 2008, de la fuente www.sloowfood.cl -), implica un ingreso total de 668.605 Euros. Considerando el precio promedio del Euro en la temporada 2007/08 de $\$ 712,71$, resultan $\$ 476.521 .470$ en moneda nacional.
Estimación de márgenes de comercialización El pescador vende a \$ 8.000, el intermediario insular \$ 9.000, el intermediario mayor a $\$ 16.500$, el comprador detallista $\$ 20.000$, el comprador extranjero $\$ 24.945$ y el comprador extranjero detallista $\$ 42.763$

A continuación, en la Tabla 6 , se presentan el precio venta $y$ el margen de comercialización de cada integrante de la cadena.

Tabla 6: Margen de Comercialización

\begin{tabular}{|c|c|c|c|c|c|c|}
\hline \multicolumn{7}{|l|}{ ESLABÓN } \\
\hline & \multicolumn{4}{|l|}{ Nacional } & \multicolumn{2}{|c|}{ Internacional } \\
\hline & Pescador & $\begin{array}{l}\text { Intermediario. } \\
\text { Insular }\end{array}$ & $\begin{array}{l}\text { Intermediario } \\
\text { Mayor }\end{array}$ & Detallista & $\begin{array}{l}\text { Comprador } \\
\text { extranjero }\end{array}$ & Detallista \\
\hline $\begin{array}{l}\text { Precio } \\
\text { Venta }\end{array}$ & 8.000 & 9.000 & 16.500 & 20.000 & 24.945 & 42.763 \\
\hline Porcentaje & 18,71 & 21,05 & 38,58 & 46,77 & 58,33 & 100,00 \\
\hline
\end{tabular}

Fuente: Elaboración Propia en base a la información de las encuestas

A continuación se expondrán los flujos de costos por eslabón de la cadena

\section{a) Primer Eslabón Pescadores}

Este análisis, se realizó comparando, costos e ingresos por cada grupo de pescador, los cuales están diferenciados por proveedores o intermediarios, (debido a que estos también participan en la cadena de comercialización, como proveedores de insumos), donde se puede observar que el grupo A3, tiene un margen de 33,8\%. Además, se visualiza que sus costos variables son menores, lo que implica que una eficiencia económica respecto a las otras agrupaciones. La tabla 7 ha sido realizada con las mismas cantidades extraídas, para cada grupo de pescadores, según intermediario I1, I2, I3 y D (directo), considerando el mismo porcentaje de mermas, basada en el promedio que estos tienen, para así hacer una comparación entre grupos de pescadores e identificar al más eficiente en costos.

b) Segundo integrante Intermediario Isla:
Se consideraron los siguientes costos relacionados con su rol, en esta cadena:

a) Costos Variables Materia Prima ( se valoró en base a una cantidad promedio mensual, noventa unidades de langostas, por grupo de 10 pescadores para cada intermediario),

Materiales de embalaje

b) Costos Fijos sigue como metodología

Mantención en viveros: se calculó teniendo en cuenta que cada intermediario tiene 5 viveros, para los cuales la mantención implica un costo mensual de $\$ 60.000$

Mano de obra: Sueldo por persona que trabajan en cada empresa

Costo de transporte isla- continente: No se valora en el caso del grupo I1, I2 y D, debido a que el intermediario continente se hace cargo de estos costos 
Tabla 7: Margen de utilidad por agrupación de pescadores por temporada

\begin{tabular}{|c|c|c|c|c|c|c|}
\hline \multicolumn{3}{|c|}{ Pescador Promedio } & \multirow{2}{*}{$\begin{array}{c}\text { Pescador Grupo } \\
\text { I3 } \\
\\
648.000\end{array}$} & \multirow{2}{*}{$\begin{array}{l}\text { Pescador } \\
\text { Grupo I2 } \\
\\
648.000\end{array}$} & \multirow{2}{*}{$\begin{array}{l}\text { Pescador } \\
\text { Grupo I1 } \\
\\
648.000\end{array}$} & \multirow{2}{*}{$\begin{array}{c}\text { Pescador } \\
\text { Directo } \\
\\
648.000\end{array}$} \\
\hline INGRESOS & $\$$ & 648.000 & & & & \\
\hline Langosta & & Unidad & & & & \\
\hline Precio Venta & \$/unidad & 8.000 & 8.000 & 8.000 & 8.000 & 8.000 \\
\hline $\begin{array}{l}\text { Cantidad } \\
\text { Mensual }\end{array}$ & Unidades & 90 & 90 & 90 & 90 & 90 \\
\hline Mermas & $\%$ & 10 & 10 & 10 & 10 & 10 \\
\hline $\begin{array}{l}\text { COSTO } \\
\text { TOTAL }\end{array}$ & $\$$ & 402.682 & $\begin{array}{c}429.142 \\
\text { COSTOS FIJOS }\end{array}$ & 623.902 & 476.572 & 439.762 \\
\hline Cuota sindical & $\$$ & 12.000 & 12.000 & 12.000 & 12.000 & 12.000 \\
\hline Mano de Obra & $\$$ & 159.000 & 159.000 & 159.000 & 159.000 & 159.000 \\
\hline $\begin{array}{l}\text { Mantención } \\
\text { embarcación }\end{array}$ & $\$$ & 36.202 & 36.202 & 36.202 & 36.202 & 36.202 \\
\hline Subtotal CF & $\$$ & 207.202 & 207.202 & 207.202 & 207.202 & 207.202 \\
\hline & & & COSTOS VARIAB & & & \\
\hline Bencina & \$/unidad & 1.827 & 1.689 & 1834 & 1889 & 1722 \\
\hline $\begin{array}{l}\text { Mantención } \\
\text { Motor }\end{array}$ & \$/unidad & 93 & 91 & 86 & 144 & 76 \\
\hline $\begin{array}{l}\text { insumos de } \\
\text { pesca }\end{array}$ & \$/unidad & 252 & 636 & 2.669 & 905 & 700 \\
\hline ropa de agua & & 44 & 50 & 41 & 55 & 86 \\
\hline $\begin{array}{l}\text { Costo por } \\
\text { Unidad }\end{array}$ & & 2.216 & 2.466 & 4.630 & 2.993 & 2.584 \\
\hline Subtotal CV & $\$$ & 195.480 & 221.940 & 416.700 & 269.370 & 232.560 \\
\hline Utilidad & $\$$ & 245.318 & 218.858 & 231.300 & 378.630 & 415.440 \\
\hline $\begin{array}{l}\text { Margen de } \\
\text { utilidad }\end{array}$ & $\%$ & 37,9 & 33,8 & 3,7 & 26,5 & 32,1 \\
\hline
\end{tabular}

Fuente: Elaboración Propia en base a la información de las encuestas

En la tabla 8, se presenta los ingresos y egresos de la comercialización que realizan los intermediarios de la
Isla, lo que permite calcular que sus utilidades ascienden a $\$ 175.770$, promedio mensual. 
Tabla 8: Cálculo de utilidad de los intermediarios de Juan Fernández en comercialización de langostas

\begin{tabular}{|c|c|c|c|}
\hline INGRESOS & $\$$ & 8.642 .700 & 15.784 .784 \\
\hline Langostas & & Unidad & Kilo \\
\hline Precio Venta & \$/unidad & 9.000 & 12.162 \\
\hline Cantidad Mensual & Unidades & 900 & 1.216 \\
\hline Mermas & $\%$ & 3,00 & 3,00 \\
\hline COSTO TOTAL & $\$$ & 7.640 .000 & 13.844 .032 \\
\hline \multicolumn{4}{|c|}{ COSTOS FIJOS } \\
\hline Viveros e instalaciones & $\$$ & 60.000 & \\
\hline Asistente Administrativa & $\$$ & 20.000 & \\
\hline Total Costos Fijos & $\$$ & 80.000 & 80.000 \\
\hline \multicolumn{4}{|c|}{ COSTOS VARIABLES } \\
\hline \multicolumn{4}{|l|}{ Langosta } \\
\hline Adquisición Materia Prima & \$/unidad & 8.000 & 10.811 \\
\hline Empaque & \$/unidad & 400 & 541 \\
\hline Costo por Unidad & & 8.400 & 11.352 \\
\hline total Costos Variables & $\$$ & 8.316 .000 & 15.188 .976 \\
\hline Utilidad antes de impuesto & $\$$ & 217.000 & 461.482 \\
\hline $\begin{array}{l}\text { Utilidad después de } \\
\text { impuesto }\end{array}$ & $\$$ & 175.770 & 373.800 \\
\hline
\end{tabular}

Fuente: Elaboración Propia en base a la información de las encuestas

c) Tercer integrante Intermediario continente

Se consideraron los siguientes costos relacionados con su rol en esta cadena:

- Costos Variables

Materia Prima (se valoró en base a una cantidad promedio mensual, noventa unidades de langostas, por un promedio de pescadores, por grupo de intermediario), transporte nacional e internacional, mantención de viveros, certificación PAC y empaque
- Costos Fijos: administración

En la tabla 9 y 10, se resumen los costos por unidad, donde se estimaron una cantidad de 900 unidades de langostas mensuales, basándose en un promedio de pescadores por grupo, con una cantidad extraída promedio mensual de noventa, en este eslabón se observa un costo $\$ 2.160 .000$ en el trasporte y una utilidad de $\$ 7.547 .530$ 
Tabla 9: Costos de intermediario Continente mercado nacional (\$)

\begin{tabular}{lc}
\hline \multicolumn{1}{c}{ INGRESOS } & 13.430 .250 \\
\hline LANGOSTAS & Unidad \\
Precio Venta CIF & 15.875 \\
Cantidad Mensual & 900 \\
Mermas & 6,00 \\
COSTO TOTAL & 10.728 .000 \\
COSTOS FIJOS & 918.000 \\
Administración & 600.000 \\
Administrador & 159.000 \\
Asistente Administrativa & 159.000 \\
Ayudante Viveros Isla & 918.000 \\
\hline Subtotal CF & \\
COSTOS VARIABLES & 8.000 \\
\hline $\begin{array}{l}\text { Adquisión Materia Prima } \\
\text { Transporte Isla - Continente }\end{array}$ & 1.800 \\
Transporte Aeródromo - Viveros & 200 \\
Mantención en Viveros & 500 \\
\hline Empaque & 400 \\
Costo por Unidad & 10.900 \\
\hline Subtotal CV & 9.810 .000 \\
\hline Utilidad antes de impuestos & 2.702 .250 \\
\hline Utilidad después de impuestos & 2.188 .823 \\
\hline Retorno sobre Ventas & 20,1 \\
\hline
\end{tabular}

Fuente: Elaboración Propia en base a la información de las encuestas 
Tabla 10 Costos de intermediario continente mercado internacional

\begin{tabular}{|c|c|c|}
\hline \multicolumn{3}{|l|}{ Intermediarios Continente } \\
\hline INGRESOS & $\$$ & 28.500 .828 \\
\hline LANGOSTAS & & Kilo \\
\hline Precio Venta CIF & \$/unidad & 24.945 \\
\hline Cantidad Mensual & unidades & 1216 \\
\hline Mermas & $\%$ & 6,04 \\
\hline COSTO TOTAL & $\$$ & 21.786 .992 \\
\hline COSTOS FIJOS & & \\
\hline Administración & $\$$ & 759000 \\
\hline Administrador & $\$$ & 600000 \\
\hline Asistente Administrativa & $\$$ & 159000 \\
\hline Ayudante Viveros Isla & $\$$ & 159000 \\
\hline Subtotal CF & $\$$ & 918.000 \\
\hline COSTOS VARIABLES & & \\
\hline Langosta & & \\
\hline Adquisión Materia Prima & \$/unidad & 10.811 \\
\hline Transporte Isla - Continente & \$/unidad & 2.432 \\
\hline Transporte Aeródromo - Viveros & \$/unidad & 270 \\
\hline Mantención en Viveros & \$/unidad & 676 \\
\hline Transporte Viveros - Planta Certificación & \$/unidad & 270 \\
\hline Certificación PAC & \$/unidad & 1.892 \\
\hline Empaque & \$/unidad & 541 \\
\hline Transporte Planta Certificación-Aeropuerto Internacional & \$/unidad & 270 \\
\hline Costo por Unidad & & 17.162 \\
\hline PRECIO CIF APROXIMADO & Por Kilo & total \\
\hline Agencia de Aduana - cargo & & 200.000 \\
\hline transporte Aéreo & 2 dolares & 1.348 .704 \\
\hline Subtotal & & 1.548 .704 \\
\hline Total Costo a Comprador Europeo & & 22.417 .696 \\
\hline COSTO TOTAL POR KILO & & 18.436 \\
\hline Subtotal CV & $\$$ & 20.868 .992 \\
\hline Utilidad antes de impuesto & $\$$ & 6.713 .836 \\
\hline Utilidad después de impuesto & $\$$ & \\
\hline Retorno sobre Ventas & $\%$ & 23,6 \\
\hline
\end{tabular}

Fuente: Elaboración Propia en base a la información de las encuesta

Análisis de Costos Variables por trampa en una temporada

Este análisis, se realiza para identificar, qué variables al aumentar sus costos tienen mayor incidencia en las utilidades. En primer lugar, se verificó la normalidad de los datos, para determinar si la distribución de la variable de respuesta (Utilidades por Trampa), es normal mediante el test de Kolmogorov-Smirnov, donde el resultado fue favorable, ya que se aceptó la hipótesis obteniendo un p-valor 0,994 mayor al 0,05, lo 
que indica que las utilidades por trampa, se distribuyen en una curva normal, en donde la media es 121.437,1 y la desviación estándar 65.193,14 (ver apéndice V). En
Figura 4, se corrobora lo anterior, al mostrar que los datos, que han sido analizados se concentran en torno a la línea recta o pendiente de la Distribución Gaussiana.

Figura 4: Distribución de las variables utilidad por trampa

\section{Variable dependiente: UTILIDAD}

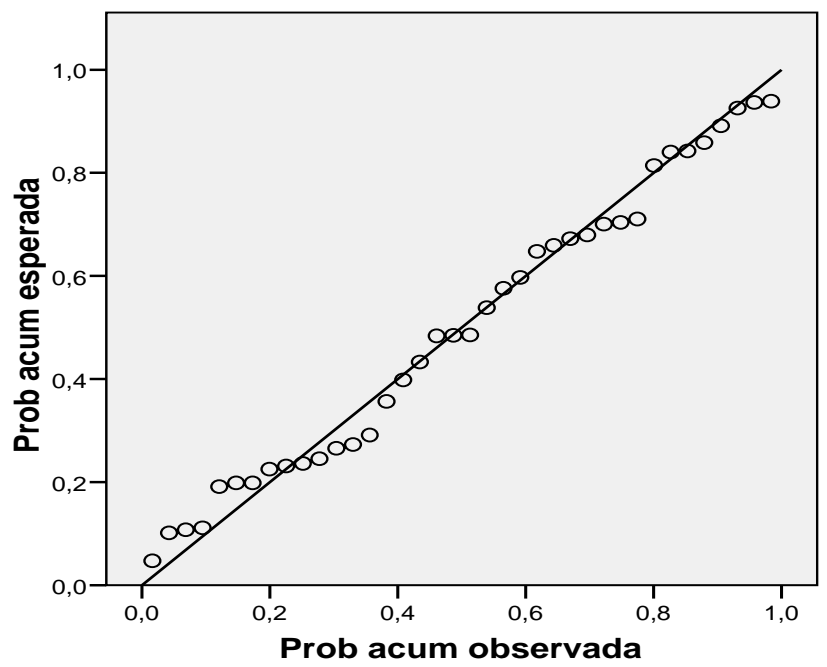

Fuente: Elaboración Propia en base a la información de las encuestas

En segundo lugar, debido a que los datos se ajustan a una distribución normal, se procedió a medir el grado de asociación lineal entre dos variables a través del Coeficiente de Correlación de Pearson. Se verifica que existe una alta correlación positiva entre las variables Bencina-Aceite y Bencina-Ropa _ agua. Por lo tanto, se hace necesario verificar, si existe Multicolinealidad, es decir, si existe una combinación lineal entre las variables explicativas, lo que provoca problemas en la precisión de los estimadores, e implica la pérdida de calidad en las predicciones, este análisis arrojó que hay valores bajos para el FIV, por lo tanto, no hay evidencia de que exista multicolinealidad (Tabla 11 ).

Tabla 11: Estadística de colinealidad (Fuente: Elaboración Propia en base a la información de las encuestas)

\begin{tabular}{|c|c|c|c|}
\hline \multirow[b]{2}{*}{ Modelo } & & \multicolumn{2}{|c|}{$\begin{array}{c}\text { Estadisticos de } \\
\text { colinealidad }\end{array}$} \\
\hline & & Tolerancia & $\mathrm{FP}$ \\
\hline \multirow[t]{11}{*}{1} & (Constante) & & \\
\hline & CORDEL & .567 & 1,765 \\
\hline & BOYAS & .792 & 1,263 \\
\hline & ANZUELO & 707 & 1,414 \\
\hline & NYLON & .766 & 1,305 \\
\hline & BENCINA & 291 & 3,442 \\
\hline & ACEITE & 503 & 1,989 \\
\hline & FILTROS & 538 & 1,858 \\
\hline & BUगAS & 662 & 1,511 \\
\hline & ROPA_AGUA & 256 & 3,900 \\
\hline & OTROS & 596 & 1,679 \\
\hline
\end{tabular}


Se realizó el análisis de Regresión, donde los coeficientes calculados que muestran mayor incidencia en las utilidades, son los de las variables: Bencinas, Bujías, Filtros y Otros, las que al incrementar sus valores inciden negativamente en la variable Utilidad.

Análisis de Márgenes
Se efectúo, a través de dos métodos, según el análisis de precio interno y retorno sobre las ventas.

Análisis de precio Interno. Se observó que el grupo con mayor margen, son los pescadores pertenecientes, al grupo A3 (Tabla 12).

Tabla 12: Análisis de precio interno por grupo de pescador

\begin{tabular}{lllllll}
\hline Pescador & & & & & \\
Análisis Precio Interno & Promedio & I3 & I2 & I1 & D \\
Precio umbral & $2.216,44$ & 2.466 & 4.630 & 2.993 & 2.584 \\
Precio técnico & 2.752 & 3.002 & 5.166 & $3.528,6$ & $3.119,6$ \\
Margen (\%) & 0,66 & 0,62 & 0,35 & 0,56 & 0,61 \\
\hline
\end{tabular}

Fuente: Elaboración Propia en base a la información de las encuestas

Análisis Retorno sobre las Ventas. Se observó, que en el grupo I3 se obtiene un mejor retorno, sobre las ventas de 33,8 $\%$ (Tabla 13)

Tabla 13: Análisis de retorno sobre las ventas por grupo de pescador

\begin{tabular}{l|c|c|l|ll}
\hline Pescador & & & & & \\
Retorno sobre Ventas (\%) & Promedio & I1 & I2 & I3 & D \\
& 37,9 & 26,5 & 3,7 & 33,8 & 32,1 \\
\hline
\end{tabular}

Fuente: Elaboración Propia en base a la información de las encuestas

\section{Conclusiones}

En la primera fase, los pescadores representan la mayor fuerza laboral de la zona, puesto que en esta localidad existe un gran número de crustáceos, octópodos, peces, entre otros, lo que permite que la economía se base en la pesca. Además, en cada familia existe uno o más pescadores, de forma hereditaria.

Son los propios pescadores, quienes venden en playa, sus capturas a intermediarios locales, quienes venden a los intermediarios continente, y estos finalmente llegan al consumidor final.

En la segunda fase, se encuentran los intermediarios, microempresarios locales, los distribuidores del mercado nacional e internacional, finalmente los consumidores.
Los distintos grupos de pescadores se reparten como proveedores de distintos intermediarios.

En la cadena de comercialización de la langosta, se evidencian altos costos de transporte, tanto nacional como internacional.

El mayor porcentaje de las ganancias de este negocio lo alcanza el eslabón del comercializador extranjero, con un $58,3 \%$, obteniendo un retorno sobre sus ventas de $24 \%$.

Los pescadores tienen un $37,9 \%$ retorno sobre sus ventas, pero sólo logran un $18,7 \%$ del valor final de este producto.

La falta de coordinación es evidente en vez de comprar los insumos por mayor, en equipo, cada cual compra por su lado y no se tiene una visualización de sus 
costos clara con respecto a quien le compran o con quien se comprometen, por lo cual el objetivo de este estudio fue describir la cadena de comercialización, donde les indica claramente que es mejor asociarse y vender su producción de manera conjunta mediante la Agrupación I3, rompiendo así las practicas de monopsonio observada en el mercado playa y de esta manera poder aumentar el precio de su producto y manejar la oferta de acuerdo a este

\section{Referencias}

1. Andrade, H. 1985. Crustáceos decapados marinos del archipiélago de Juan Fernández, En: Investigaciones marinas en el archipiélago de Juan Fernández, P. Arana (ed.), Escuela de Ciencias del Mar, UCV. Valparaíso, 109-116

2. Arana, P. 2000. Pesca exploratoria con trampas alrededor de las islas Robinson Crusoe y Santa Clara, archipiélago de Juan Fernández, Chile. Investigaciones del Mar, UCV, Valparaíso, 39-52

3. Arana, P. \& R. Vega. 2000. Esfuerzo, captura y captura por unidad de esfuerzo en la pesquería de la langosta de Juan Fernández (Jasus frontalis) durante la temporada de pesca 1996-1997.Invest. Mar., Valparaíso

4. FIP, 2004-48. Monitoreo biológico-pesquero de la langosta y cangrejo dorado en el archipiélago de Juan Fernández, Escuela de ciencias del mar, UCV.

5. PLADECO (2007) plan de desarrollo comunal para la ilustre municipalidad de Juan Fernández. Gobierno de la V región, Chile. 